\title{
PERSONALISED RECOMMENDER ENGINE USING A PROBABLISTIC MODEL
}

\author{
Ajay Rajendra Dhruv \\ Research Scholar, epartment of Information Technology, Vidyalankar Institute of \\ Technology, Mumbai, India \\ Dr. J W Bakal \\ Principal \\ SJCOE \\ Mumbai, India
}

\begin{abstract}
The world of e-commerce and e-business has opened many horizons to explore customers on web. Consumers are expecting businesses to approach and please them with their expectations. This has given rise to recommender systems. Many of the recommender systems are generalized in nature which are often based on market stratum and user predictions. However, extensive research is being carried out in providing personalized recommendations using association rules, customer segmentation, social media ontologies and demographics. There are many issues in the implementation of these systems. This paper discusses diverse recommender approaches proposed in the past with a comparative study and gap analysis. It also proposes a Hybrid Personalized Recommender system by using a probabilistic model.
\end{abstract}

Keyword head - Recommender systems

Cite this Article: Ajay Rajendra Dhruv and Dr. J W Bakal, Personalised Recommender Engine Using a Probablistic Model, International Journal of Computer Engineering and Technology, 10(2), 2019, pp. 41-48.

http://iaeme.com/Home/issue/IJCET?Volume=10\&Issue=2

\section{INTRODUCTION}

There has been an enormous growth in the number of products sold online which has sometimes become difficult for the users to decide which products to buy and which products not to buy. In such a confusion, recommender systems provide ease to the consumer market. Recommender systems are aimed at providing generalised or personalised recommendations to the user for better decision making. Recommender systems are built by keeping in mind various parameters such as usability, serendipity, accuracy, novelty etc. The section below describes various approaches of recommender systems that are widely used. 


\subsection{Recommendation system approaches}

Recommendation systems work by predicting the relevance of items for users by analyzing the users' behavior, browsing history, ratings, interaction with items, demography or other information that can learn the system about the users and the items. This can be done in many different ways, with collaborative filtering and content-based filtering as the two main approaches (Bari, Chaouchi, and Jung 2014). Other important approaches are demographicbased, knowledge-based, community-based and hybrid approaches (Ricci, Rokach, and Shapira 2015). Each of these approaches will be presented here

\subsubsection{Collaborative Filtering}

Collaborative filtering is an approach used to make personalized recommendations that are based on patterns of ratings and usage by the users of a system (Koren and Bell 2011). The idea behind this approach is that if a group of users share opinion on a set of topics, they may also share opinion on another topic (Bari, Chaouchi, and Jung 2014). The system collects large amounts of data, and analyses it to find latent factors or similarities between users or between items.

\subsubsection{Content Based}

Content-based filtering methods are based on a description of the item and a profile of the user's preferences. In a content-based recommender system, keywords are used to describe the items and a user profile is built to indicate the type of item this user likes. In other words, these algorithms try to recommend items that are similar to those that a user liked in the past (or is examining in the present). In particular, various candidate items are compared with items previously rated by the user and the best-matching items are recommended. This approach has its roots in information retrieval and information filtering research.

\subsubsection{Demographic Based}

Recommendation systems can make recommendations based on demographic information about the users. The idea is that users with different demographic features like age, gender, Language, nationality and education will have different interests, and then should get recommendations accordingly. In demographic-based recommendation systems, users are categorized in terms of their personal attributes and the recommendations are made based on demographic classes (Burke 2002). For example, Robert who is a 20 -year-old Canadian man, will be categorized in a group of persons with the same characteristics, and the items recommended to him will be the items that are most preferred by the other members of the group.

\subsubsection{Knowledge Based}

Knowledge-based recommendation systems give recommendations based on domain knowledge about how different item features meet user needs and how items are useful for the user (Ricci, Rokach, and Shapira 2015). They do not try to make any long-term generalizations about the users, but instead base the suggestions on an evaluation of the match between a user's need and the options available (Burke 2002). For example, if John is going to watch a movie together with his little sister, who is nine years old, he will look for a different type of movie than what he usually likes. Therefore, it is better that the recommendations he gets from the system are based on the actual need in this situation, rather than on his usual preferences. With a knowledge-based system, John can specify together with his sister which features they would like the movie to have, e.g., "maximum 1 hour" and "children's movie", and the system will find the movies that best fit their needs. 


\subsubsection{Community Based}

Recommendation systems that are community-based gives recommendations based on the likings and preferences of a user's social connections (Ricci, Rokach, and Shapira 2015). This builds on people's tendency to prefer recommendations by friends compared to those from an online system (Sinha and Swearingen 2001). The idea is to utilize the ratings from friends to make recommendations that are as good as if they were given by friends. This can be done by collecting information about the user's social relations at social networks and then recommending items highly rated by the user's social community (Sahebi and Cohen 2011). For example, if a high proportion of John's friends on Facebook like the same movie, he can be recommended this movie.

\subsubsection{Combination Based}

Combination recommendation systems combine two or more approaches discussed above to reduce the drawbacks of each individual approach, and by this getting an improved performance (Burke 2002). For example, collaborative filtering, which in general has good performance, but suffers from the cold start problem, can be combined with an approach that does not have this problem, like the content-based approach. Several methods can be used to make a hybrid recommendation system. The approaches can be implemented separately and combine the results from each, some parts of one approach can be utilized in another approach or a unified recommendation system can be made by bringing together the different approaches (Isinkaye, Folajimi, and Ojokoh 2015).

\section{RELATED WORK}

There are various recommendation system approaches and combinations of these can be used. To illustrate how recommendation systems function in real life, some recommendation systems used by large companies will be presented here. However, these companies have business secrets, so the presentation of the recommendation systems is based on articles and public information about their recommendation systems and may have changed from the publication of this information.

\subsection{Recommendation systems in practice}

\subsubsection{Amazon}

The American e-commerce company Amazon (amazon.com) was one of the first companies to use recommendations in a commercial setting. They are famous for recommendations like "Customers who bought this item also bought..." and "frequently bought together". Amazon bases its recommendations on buying behavior, explicit ratings on a scale from 1 to 5 and browsing behavior (Aggarwal 2016, 5). Linden, Smith, and York (2003) explains how Amazon uses an item-based collaborative filtering approach to recommend products to its customers. The algorithm builds a similar items table by finding items often bought together. This is done by iterating over all the items in the product catalogue, and for each customer who bought it, record that this item is bought together with each of the other items bought by this customer. Then, similarity is computed between all pairs of items collected, typically done by a cosine measure. This calculation is made offline, so the most similar products from the similar-items table can be presented fast to the user.

\subsubsection{Netflix}

Netflix (netflix.com) is a company that provides streaming of movies and series. It offers its customers a personalized interface, where previous views, ratings and items added to the user's 
list give basis for the titles presented to the user. Netflix typically recommends a set of videos in a genre or a set of videos based on a user's interaction with an item. The recommendations are then justified by what the set is based on, as "Because you watched ...", "Comedies" or "Top list for you". Each set is presented as a horizontal list of items and the user is presented to several rows of such sets. The recommendation algorithm uses a set of factors to make its recommendations. Which genres the available movies and series have, the user's streaming and rating history and all ratings made by users with similar tastes, are factors that affect the recommendations a user gets (Netflix 2016). This is an example of a hybrid recommendation system, that uses both collaborative filtering - as similar users' ratings are used to recommend, and content-based filtering techniques - as genres are used to recommend. The rating scale in Netflix is, as in Amazon, from 1 to 5 stars.

\subsubsection{Facebook}

The social networking site Facebook (facebook.com) makes recommendations to its users at multiple areas of the website. Recommendation systems are used to suggest new friends, choose which posts should be showed at the top of a user's newsfeed, propose pages for a user to like and recommend apps to download. The algorithm used for recommending apps in Facebook's app center will here be presented to give an understanding of how Facebook recommends content to its users. The recommendation system used in Facebook's app center has three major elements (Facebook Code 2012). The first is candidate selection, where a number of promising apps are selected. This selection is based on demographic information, social data and the user's history of interaction and liking of items. The second element in the recommendation system is scoring and ranking. Explicit features like demographic data and dynamic features like number of likes are important when the ranking scores for the apps are calculated, but the most important feature is learned latent features. This is features learned from the user's history of interaction with items. The predicted response for a user to an object, is calculated by the dotproduct of two vectors, where one is the latent features of the user and the other is for the characteristic of the object. The last element of the recommendation system is real time updates. With a huge number of users and new apps coming in frequent, the indexes and latent features must be updated in real-time to ensure the best possible recommendations. This is a good example of a model-based latent factor model that also utilizes the demographic - and community-based approach. Figure 5 shows recommendations of games in the app centre. Facebook is known for its like-rating, but uses also ratings on a scale from 1 to 5 .

\subsubsection{YouTube}

The world's most popular online video community, YouTube (youtube.com), gives personalized recommendations of videos to its users, with a goal of letting the users be entertained by content they find interesting (Davidson et al. 2010). An important part in YouTube's recommendation system is to find the most similar videos for each video. This is done in a similar fashion to the item-based approach to collaborative filtering used by Amazon. Two videos are regarded as similar if they have a high co-visitation count, i.e., if they are watched together by the same user within a given period, typically 24 hours. This number is then normalized with a function that takes the video's global popularity into account, to avoid that the most watched videos get an advantage over the less popular ones. A mapping is then made between each video and its $\mathrm{N}$ most similar videos. To select the recommendation candidates for a user, a seed set of videos are generated. This is all the videos the user has liked explicitly or implicitly. A candidate set of videos are generated by taking the union of all videos that are like the videos in the seed set. The candidate set is then extended with all videos like the videos in the set, and this is repeated several times to increase the span of the videos. YouTube wants the recommendations to help the users to explore new content, and then it is 
important that not all videos are too like videos in the seed set. The videos in the candidate set are then ranked according to video quality and user specificity. Video quality is computed by variables independent of the user, like total view count and the total number of positive ratings for a video. YouTube has explicit data in form of likes and dislikes, and implicit data from for example viewing history, comments and sharing of videos. To ensure the relevance of the video for the user, the user specificity reflects if the video is closely matched with the user's unique taste and preferences. In the end, not only the videos that are ranked highest are recommended. Videos from different categories are selected to increase the diversity of the recommendations.

Various Recommender Engines are studied and presented in table 2.1. Gap Analysis for same is done.

Table 1 Comparative Study and Gap Analysis of various recommender systems

\begin{tabular}{|c|c|c|}
\hline $\begin{array}{l}\text { Ref } \\
\text { No. }\end{array}$ & Description & Gap Analysis \\
\hline$\left[\begin{array}{l}{[1]} \\
{[3]}\end{array}\right.$ & $\begin{array}{l}\text { A keyword-aware service recommendation method named } \\
\text { KASR is proposed in which keywords are used to indicate } \\
\text { user's preferences, and a user based Collaborative Filtering } \\
\text { algorithm is adopted to generate appropriate } \\
\text { recommendations. More specifically, keyword- candidate } \\
\text { list and domain thesauruses are provided to help obtain } \\
\text { users preferences. The active user gives his/her preferences } \\
\text { by selecting the keywords from the keyword candidate list, } \\
\text { and the preferences of the previous users can be extracted } \\
\text { from their reviews for services according to the keyword- } \\
\text { candidate list and domain thesaurus. Moreover, to improve } \\
\text { the scalability and efficiency of KASR in "Big Data" } \\
\text { environment, the system is implemented on a MapReduce } \\
\text { framework in Hadoop Platform. }\end{array}$ & $\begin{array}{l}\text { The system does not produce } \\
\text { accurate results when a term } \\
\text { appears in different categories } \\
\text { of a domain thesaurus from } \\
\text { context. } \\
\text { Less accurate predictions as it } \\
\text { does not distinguish positive } \\
\text { and negative preferences of } \\
\text { users from their reviews. }\end{array}$ \\
\hline $\begin{array}{l}{[2][11]} \\
{[17]} \\
{[18]}\end{array}$ & $\begin{array}{l}\text { A generic approach is presented for performing } \\
\text { collaborative filtering using data mining techniques to } \\
\text { discover relationships among users and items. Pearson } \\
\text { correlation method is used as a similarity metric. The } \\
\text { system is implemented in Apache Hadoop and Mahout for } \\
\text { user and item-based recommendations. The system is } \\
\text { evaluated using two parameters, Precision and Recall. The } \\
\text { implementation framework indicates that the use of } \\
\text { Apache Hadoop with Mahout is most suitable for } \\
\text { implementation of large-scale systems. }\end{array}$ & $\begin{array}{l}\text { The system does not scale to } \\
\text { Prescriptive Analytics. } \\
\text { Consumer preferences are } \\
\text { minimum }\end{array}$ \\
\hline
\end{tabular}




\begin{tabular}{|c|c|c|}
\hline [4] & $\begin{array}{l}\text { A prescriptive analytics system, InSciTe advisory is } \\
\text { developed to provide researchers with advice of their } \\
\text { future research direction and strategy. The system analyses } \\
\text { several thousands of heterogeneous types of data sources } \\
\text { such as papers, patents, reports, web news, web magazines, } \\
\text { and collective Intelligence data. It consists of two main } \\
\text { parts, descriptive analytics and prescriptive analytics. Once } \\
\text { given a researcher, the descriptive analytics part provides } \\
\text { results from activity history and research power w.r.t. the } \\
\text { designated researcher. Then, prescriptive analytics part } \\
\text { suggests a group of role model researchers to the } \\
\text { researcher, as well as how to be like the role model } \\
\text { researchers. }\end{array}$ & $\begin{array}{l}\text { Feedback from the researcher is } \\
\text { not taken to improvise the } \\
\text { recommendations }\end{array}$ \\
\hline$[5][12]$ & $\begin{array}{l}\text { A case-based reasoning system is proposed to identify and } \\
\text { recommend the items that seem more suitable for } \\
\text { completing a user's buying experience provided that he or } \\
\text { she has already selected some items. The system models } \\
\text { complete transactions as cases and recommended items } \\
\text { come from the evaluation of those transactions. Because } \\
\text { the cases are not restricted to the user who purchased } \\
\text { them, the developed system can generate accurate item } \\
\text { recommendations for joint item selections, both for new } \\
\text { and existing users. The system considers only the presence } \\
\text { of items in the user profiles without evaluating the items' } \\
\text { co-occurrences within transactions. }\end{array}$ & $\begin{array}{l}\text { Co-occurrence of an item in a } \\
\text { transaction is not considered as } \\
\text { it could help in giving } \\
\text { meaningful predictions. } \\
\text { User demographic is not } \\
\text { considered as an input for } \\
\text { recommending preferences. }\end{array}$ \\
\hline$[6][10]$ & $\begin{array}{l}\text { A cross platform multi- modal probabilistic model is } \\
\text { proposed to address the inter platform recommendation. A } \\
\text { recommendation on two platforms Flickr and Foursquare } \\
\text { has been conducted where photo recommendations are } \\
\text { given from Flickr to Foursquare users and venue } \\
\text { recommendations are given from Foursquare to Flickr } \\
\text { users. }\end{array}$ & $\begin{array}{l}\text { New users are not considered in } \\
\text { the proposed system. }\end{array}$ \\
\hline [7] & $\begin{array}{l}\text { A neighbour diversification algorithm is proposed to get a } \\
\text { diverse neighbour set and then provide recommendations } \\
\text { based on this neighbour set. The system improves overall } \\
\text { novelty, coverage and accuracy. }\end{array}$ & $\begin{array}{l}\text { Behavioural patterns of the } \\
\text { users are not taken into } \\
\text { consideration. }\end{array}$ \\
\hline $\begin{array}{l}{[8]} \\
{[13]} \\
{[4]}\end{array}$ & $\begin{array}{l}\text { The proposed system improvises Markov chain based } \\
\text { recommender model by using a hybrid approach that } \\
\text { provides good accuracy as well as resolves the diversity- } \\
\text { accuracy dilemma. An accurate recommender system } \\
\text { would provide suggestions based on the users action but } \\
\text { the proposed model takes care of novelty and diversity of } \\
\text { items by providing suggestions for items that the users } \\
\text { would not discover by themselves. }\end{array}$ & $\begin{array}{l}\text { Depedence of performance } \\
\text { measure is on ' } \alpha \text { ' }\end{array}$ \\
\hline
\end{tabular}

\section{Proposed Work}

Figure 1 indicates the proposed model. Initially there are two inputs i.e consumer data and product data. Consumer data consists of products and its frequency, demographics and their past transactions. Product data consists of trending products in the market an new products 
launched. Using these two primary inputs, a probablistic engine is developed by forming a product matrix for every user and assigning weights to the product category. Multiply the probabilities of products with the category weights to get the product score matrix MPS. . Based on the Recency - Frequency - Monetary (RFM) values and consumer demographics, derive ' $\alpha$ ' for every product which will be multiplied to the product score to generate optimised product score matrix MOPS. The optimised product score will imply the popularity of the product w.r.t. particular consumer. Based on the optimised score based predictions, recent, trending and new products and previous recommendation and feedback - generate recommendations.

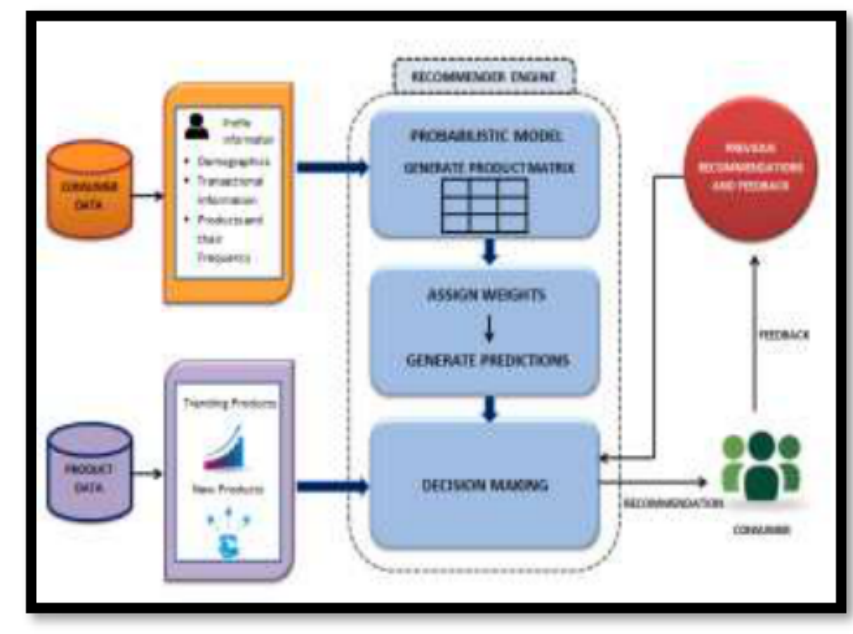

Figure 1. Probabilistic Model based Recommender Engine

\section{ConClusion}

The e-commerce industry requires a very accurate recommender system which not only recommends which products to buy but also understand the customer preferences with scale of time. The paper attempts to propose a model which covers all dimensions of user behavior for suggesting the best recommendations.

\section{REFERENCES}

[1] Shunmei Meng, Wanchun Dou, Xuyun Zhang, and Jinjun Chen, Senior Member, IEEE, "KASR: A Keyword-Aware Service Recommendation Method on MapReduce for Big Data Applications", IEEE Transactions on Parallel and Distributed Systems, vol. 25, no. 12, December 2014.

[2] Lavannya Bhatia, S.S.Prasad, "Building a Distributed Generic Recommender using Scalable Data Mining Library", IEEE International Conference on Computational Intelligence \& Communication Technology, 2015.

[3] Jinhong Kim, Sung- Tae Hwang, "A study of conceptual Recommender System for Big Data Platform", 8th International Conference on Database Theory and Application, 2015.

[4] Sa-kwang Song, Donald J. Kim, Myunggwon Hwang, Jangwon Kim, Do-Heon Jeong, Seungwoo Lee, Hanmin Jung, Wonkyung Sung, "Prescriptive Analytics for Improving Research Power", IEEE 16th International Conference on Computational Science and Engineering, 2013.

[5] Anna Gatzioura, Miquel Sànchez-Marrè, "A Case-Based Recommendation Approach for Market Basket Data", IEEE Intelligent systems, 2015.

[6] Weiqing Min,Bing-Kun Bao, Changsheng Xu, M.Shamim Hossain, “ Cross - Platform Multi Modal Topic Modelling for Personalized Inter - Platform Recommendation”, IEEE Transactions on Multimedia, vol.17, No.10, October 2015. 
[7] Chao Yang, Cong Cong Ai, Renfa Li, "Neighbor Diversification - Based Collaborative Filtering for Improving Recommendation Lists", IEEE International Conference on High Performance Computing and Communications, 2013.

[8] Amin Javari, Mahdi Jalili, "A Probabilistic model to resolve diversity-accuracy challenge of recommendation systems", Knowledge and Information Systems, Springer 2014.

[9] Haitao $\mathrm{Wu}$, Wen-Kuang Chou, Ningbo Hao, Duan Wang and Jingfu Li, "Collaborative filtering recommendation based on conditional probability and weight adjusting", International Journal of Computational Science and Engineering, Vol. 10, Nos. 1/2, 2015.

[10] Y. Chen, A. Cheng, and W. Hsu, "Travel Recommendation by Mining People Attributes and Travel Group Types from Community- Contributed Photos", IEEE Transactions Multimedia, vol. 25, no. 6, pp. 1283-1295, Oct. 2013.

[11] Negar Hariri, "Supporting Domain Analysis through Mining and Recommending features from Online Product Listings", IEEE Transactions on Software Engineering, Vol.39, No.12, Dec 2013, pp.73-82.

[12] Fong, "Web Content Recommender System based on Consumer Behaviour Modelling", IEEE Transactions on Consumer Electronics, Vol.57, No.2, May 2011.

[13] Jia Hao, Yan Yan, Guoxin Wang, Lin Gong, and Bo Zhao, "A Probability-Based Hybrid User Model for Recommendation System", Mathematical Problems in Engineering Volume 2016 (2016), Article ID 9535808.

[14] Ming Li and Benjamin Dias, Wael El-Deredy, Paulo J. G. Lisboa, "A Probabilistic Model for Item-Based Recommender Systems”, ACM 978-1-59593-730-8/07/0010, 2007.

[15] ] Gediminas Adomavicius, Bamshad Mobasher, Francesco Ricci, and Alex Tuzhilin, "Context-Aware Recommender Systems", Association for the Advancement of Artificial Intelligence, 2011.

[16] Soanpet Sree Lakshmi, Dr.T.Adi Lakshmi, "Recommendation Systems: Issues and challenges", International Journal of Computer Science and Information Technologies, Vol. 5 (4), 5771-5772, 2014.

[17] Shikha Agarwal, Archana Singhal, "Ontology Enrichment: A Tool to Empower Hybrid Recommender Systems", International Journal of Recent Trends in Engineering \& Technology, Vol. 11, June 2014.

[18] Badrul Sarwar, George Karypis, Joseph Konstan, and John Riedl, "Item-Based Collaborative Filtering Recommendation Algorithms", ACM 1-58113-3480/01/0005,2001.

[19] Ching-Hsue Cheng, You-Shyang Chen, "Classifying the segmentation of customer value via RFM model and RS theory", Expert Systems with Applications: An International Journal,Volume 36 Issue 3, April, 2009.

[20] Shunmei Meng, Wanchun Dou, Xuyun Zhang, and Jinjun Chen, Senior Member, IEEE, "KASR: A Keyword-Aware Service Recommendation Method on MapReduce for Big Data Applications", IEEE Transactions on Parallel and Distributed Systems, vol. 25, no. 12, December 2014.

[21] Lavannya Bhatia, S.S.Prasad, "Building a Distributed Generic Recommender using Scalable Data Mining Library”, IEEE International Conference on Computational Intelligence \& Communication Technology, 2015.

[22] Jinhong Kim, Sung- Tae Hwang, "A study of conceptual Recommender System for Big Data Platform", 8th International Conference on Database Theory and Application, 2015. 